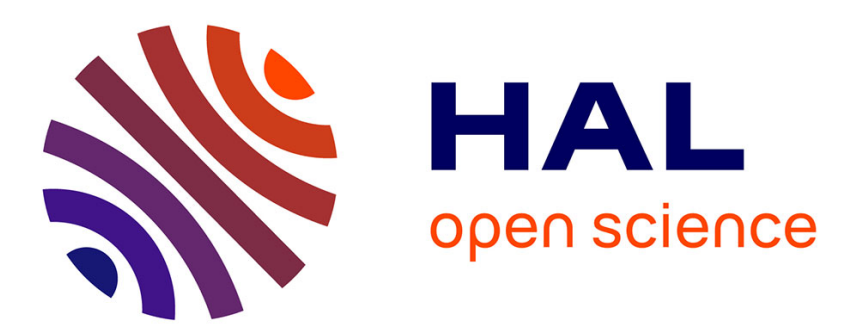

\title{
Potential of fluorescence spectroscopy to predict fatty acid composition of beef
}

\author{
A Aït-Kaddour, Agnès Thomas, J Mardon, S Jacquot, Anne Ferlay, \\ Dominique Gruffat
}

\section{- To cite this version:}

A Aït-Kaddour, Agnès Thomas, J Mardon, S Jacquot, Anne Ferlay, et al.. Potential of fluorescence spectroscopy to predict fatty acid composition of beef. Meat Science, 2016, 113, pp.124-131. 10.1016/j.meatsci.2015.11.020 . hal-02636973

\section{HAL Id: hal-02636973 \\ https://hal.inrae.fr/hal-02636973}

Submitted on 27 May 2020

HAL is a multi-disciplinary open access archive for the deposit and dissemination of scientific research documents, whether they are published or not. The documents may come from teaching and research institutions in France or abroad, or from public or private research centers.
L'archive ouverte pluridisciplinaire HAL, est destinée au dépôt et à la diffusion de documents scientifiques de niveau recherche, publiés ou non, émanant des établissements d'enseignement et de recherche français ou étrangers, des laboratoires publics ou privés. 


\title{
Potential of fluorescence spectroscopy to predict fatty acid composition of beef
}

\author{
A. Aït-Kaddour a , A. Thomas s,c,d , J. Mardon ${ }^{\text {a }}$, S. Jacquot ${ }^{\mathrm{a}}$, A. Ferlay b,c,d , D. Gruffat ${ }^{\mathrm{b}, \mathrm{c}, \mathrm{d}}$ \\ a Clermont University, VetAgro Sup, UPSP no. 2011-03-100, CALITYSS, BP 10448, F-63000 Clermont-Ferrand, France \\ b National Institute for Agronomic Research (INRA), UMR 1213 Herbivores, Research Centre Auvergne-Rhône-Alpes, F-63122 Saint-Genès-Champanelle, France \\ c Clermont Université, VetAgro Sup, UMR1213 Herbivores, BP 10448, F-63000 Clermont-Ferrand, France \\ d Université de Lyon, VetAgro Sup, UMR1213 Herbivores, F-69280 Marcy l'Etoile, France
}

\section{A R T I C L E I N F O}

\section{Article history:}

Received 10 February 2015

Received in revised form 12 November 2015

Accepted 23 November 2015

Available online 24 November 2015

\section{Keywords:}

Fluorescence spectroscopy

Meat

Fatty acids

\begin{abstract}
A B S T R A C T
The present study aimed to evaluate and compare the ability of front face (FFFS) and synchronous fluorescence spectroscopy (SFS) to predict total fat and FA composition of beef LT muscles coming from 36 animals of 3 breeds (Angus, Limousin and Blond d'Aquitaine). The regression models were performed by using Partial Least Square (PLS) method. In spite of the low number of samples used, the results of this preliminary study demonstrated the ability of fluorescence spectroscopy to predict meat lipids. Nonetheless, the results suggested that the fluorescence spectroscopy is more suited to measure SFA $\left(R^{2} p \geq 0.66\right.$; RPD $\left.\geq 2.29\right)$ and MUFA $\left(R^{2} p \geq 0.48\right.$; RPD $\left.\geq 1.49\right)$ than PUFA $\left(R^{2} p \leq 0.48\right.$; RPD $\left.\leq 1.63\right)$. Moreover, $R^{2}$ and RPD factors obtained with FFFS were greater compared to the ones obtained with SFS suggesting that FFFS is more adapted to measure lipid composition of beef meat.
\end{abstract}

(C) 2015 Elsevier Ltd. All rights reserved.

\section{Introduction}

Fat and fatty acids (FAs) are major contributors of meat nutritional value. Indeed, numerous studies have highlighted the link between lipid intake and potential health effects in humans (Hooper, Abdelhamid, Moore, Douthwaite, \& Murray, 2012; Riccardi, Giacco, \& Rivellese, 2004; Wahrburg, 2004). Even if the issue remains controversial (McAfee et al., 2010), most of the dietary recommendations advise to lower the contribution of fat and saturated FA (SFA) to daily energy intakes while increasing the intake of omega-3 polyunsaturated FA (PUFA) to reduce the risk of cardiovascular disease (Gidding et al., 2005; Siri-tarino, Sun, Hu, \& Krauss, 2010a, 2010b). Furthermore, some trans-fatty acids (C18:1 trans-11) present within meat were shown to have no cholesterol-raising effect (Chardigny et al., 2008). There is also emerging evidence that conjugated linoleic acid (CLA) isomer cis-9 trans-11 is associated with prevailing beneficial health effects for humans (Benjamin \& Spener, 2009).

Thus, several strategies, mainly modulating genetics and dietary factors, are currently investigated to enhance meat FA composition (Scollan et al., 2014; Wood et al., 2008). To support this effort, fluorescence analysis was suggested to be a rapid and efficient tool useful in the quality estimation of meat products (Karoui \& Blecker, 2010; Swatland, 1987). Front-face fluorescence spectroscopy (FFFS) notably allows the investigation of solid food samples in the wavelength range of 200-750 nm. In this case, excitation and emission spectra are

E-mail address: abderrahmane.aitkaddour@vetagro-sup.fr (A. Aït-Kaddour). acquired at fixed wavelength determined as a function of the fluorophores present in the analyzed food matrix, namely tryptophan, vitamin A, riboflavin, lipid oxidation products and NADH in meat products. In a variant acquisition mode, synchronous fluorescence spectroscopy (SFS), spectra results from simultaneously scanning both the excitation and emission monochromators keeping a fixed wavelength interval between them (Karoui \& Blecker, 2010). Firstly initiated by Lloyd (1971), SFS allows the consideration of the whole fluorescence landscape meaning it retains information related to several fluorophores of the food matrix. Therefore, it could be more suitable for the analysis of complex multi-component samples as compared to conventional FFFS. Fluorescence spectroscopy has been mainly used to characterize quality traits of meat products, such as sensory properties (Dufour \& Frencia, 2001; Olsen et al., 2005; Swatland, Gullett, Hore, \& Buttenham, 1995). Extensive works using fluorescence spectroscopy associated with chemometrics were also carried out to authenticate and classify meat products as a function of their rearing conditions (Gatellier et al., 2007), manufacturing process, storage and cooking conditions (Gatellier, Santé-Lhoutellier, Portanguen, \& Kondjoyan, 2009; Møller, Parolari, Gabba, Christensen, \& Skibsted, 2003) or microbial spoilage (AïtKaddour, Boubellouta, \& Chevallier, 2011). Quantitative evaluations of some meat components, namely collagen and heterocyclic aromatic amine contents, were developed using multivariate regression techniques in meat products (Egelandsdal, Dingstad, Tøgersen, Lundby, \& Langsrud, 2005; Sahar, Portanguen, Kondjoyan, \& Dufour, 2010; Wold, Kvaal, \& Egelandsdal, 1999). However, very few data is currently available regarding the feasibility of using fluorescence spectroscopy to evaluate total fat content in meat. Wold and collaborators investigated the ability of FFFS 
to determine fat content in beef and exhibited fair models of cross validated prediction $\left(\mathrm{RMSECV}=1.89 \%, \mathrm{R}^{2}=0.71\right.$ and $\mathrm{RMSECV}=$ $1.10 \%, R^{2}=0.71$, respectively) (Wold, Kvaal et al., 1999; Wold, Lundby, \& Egelandsdal, 1999). Another work reported poor prediction models $\left(R^{2}=0.57\right)$ when testing the potential of fluorescence spectroscopy to evaluate intramuscular fat content in porcine meat (Brondum et al., 2000). Surprisingly, to our knowledge, no work investigating the potential interest of fluorescence spectroscopy in assessing fatty acid composition of meat was reported so far. Yet, an advantage of fluorescence detection compared to absorption measurements (e.g., IR) is the greater sensitivity (100-1000 times) achievable, meaning that fluorescent compounds can be investigated at very low concentration levels (in the parts per billion range).

It would be therefore interesting to determine in what extent fluorescence spectroscopy would be useful to predict the specific FA composition of meat. Similar approaches were carried out using near infrared reflectance spectroscopy. Globally, this tool appeared to accurately predict the concentration of some prominent FA and FA groups such as saturated, branched and monounsaturated FA in beef, poultry and lamb meat (Guy, Prache, Thomas, Bauchart, \& Andueza, 2011; Prieto et al., 2014; Sierra et al., 2008).

Thus, the purpose of this study is to evaluate and compare the ability of front face and synchronous fluorescence spectroscopy to predict total fat and FA composition of beef.

\section{Materials and methods}

\subsection{Animals and meat samples}

The muscle samples came from 36 bulls of 3 genotypes with varying lipogenesis capacities (Angus $>$ Limousin $>$ Blond d'Aquitaine). All the details on the experimental design and diets for the experiment were previously described by Gruffat et al. (2013). At the end of the experimental period, the bulls were slaughtered in the experimental abattoir of INRA (Saint-Genès-Champanelle, France). Samples ( 100 g) of the Longissimus thoracis (LT) muscle of the 36 animals were collected at $24 \mathrm{~h}$ post mortem from the 10th thoracic rib on the right side of the ribbed carcass. Muscle samples were cut into small cubes $\left(1 \mathrm{~cm}^{3}\right)$, immediately frozen in liquid nitrogen and stored at $-80^{\circ} \mathrm{C}$. Just before analysis, the frozen samples of the LT muscle were ground into fine and homogeneous powders in liquid nitrogen with a mixer mill (Retch MM 301, Hann Germany).

\subsection{Fatty acid analysis by gas liquid chromatography}

Total lipids were extracted according to the method of Folch, Lees, and Sloane (1957) by mixing the LT muscle powder with a 2/1 chloroform/methanol mixture ( $\mathrm{vol} / \mathrm{vol}$ ) and quantified by gravimetry. Fatty acid extraction and transmethylation into fatty acid methyl esters (FAME) were subsequently performed according to the method of Bauchart, Gladine, Gruffat, Leloutre, and Durand (2005). Fatty acid methyl ester analysis was performed with Gas Liquid Chromatography (GLC) using a Peri 2100-chromatography system (Perichrom Society, Saulx-les-Chartreux, France) fitted with a CP-Sil 88 glass capillary column (Varian, Palo Alto, CA; length $=100 \mathrm{~m}$; diam. $=0.25 \mathrm{~mm}$ ). The carrier gas was $\mathrm{H}_{2}$ and the oven and flame ionization detector temperatures described by Scislowski, Durand, Gruffat, and Bauchart (2004) were used. Total FAs were quantified using C19:0 as an internal standard. The identification of each individual FAME and the calculation of the response coefficients for each individual FAME were performed using the quantitative mix C4-C24 FAME (Supelco, Bellafonte, PA).

\subsection{Fluorescence spectroscopy}

Samples fluorescence came from the same powder as that used for fatty acid analysis (i.e. LT muscle collected at $24 \mathrm{~h}$ post mortem, immediately frozen in liquid nitrogen and stored at $-80^{\circ} \mathrm{C}$ ). Frozen samples were ground into fine and homogeneous powders in liquid nitrogen and stored at $-80{ }^{\circ} \mathrm{C}$ until analyses. Before acquisitions, the homogeneous powder of ground LT muscles was thawed during $1 \mathrm{~h} 00$ at $20^{\circ} \mathrm{C}$. Then a proportion $3 \mathrm{~g}$ of meat powder was placed between a powder sample holder and a quartz cell and mounted in a solid sample holder. Before fluorescence acquisition the sample was visually controlled to ensure that all the measurement window was totally covered with meat sample. Fluorescence spectra were recorded in two excitation modes successively on the same sample, classical excitation mode (FFFS: Front Face Fluorescence Spectra) and synchronous excitation mode (SFS: Synchronous Fluorescence Spectra) by using a FluoroMax-4 spectrofluorometer (Jobin-Yvon, Longjumeau, France) equipped with a solid sample holder with an incidence angle of the excitation radiation set at $60^{\circ}$ in order to minimize reflected light, scattered radiation and depolarization phenomena. For SFS, the excitation wavelength $\left(\lambda_{\mathrm{ex}}\right)$ and emission wavelength $\left(\lambda_{\mathrm{em}}\right)$ are scanned simultaneously (synchronously), usually maintaining a constant wavelength interval, named offset or $\Delta \lambda$, between $\lambda_{\text {ex }}$ and $\lambda_{\text {em }}$. In this study, six $\Delta \lambda(20,40,60,80,100$, and $120 \mathrm{~nm}$ ) were used. All the excitation fluorescence spectra were recorded between 250 and $550 \mathrm{~nm}$.

For FFFS, the emission spectra were recorded at 305-400, 340-540, 360-570, 400-650 and 410-700 $\mathrm{nm}$ after excitation at 290, 322, 335, 350 and $382 \mathrm{~nm}$ respectively. Those excitation spectra mainly addressed the fluorescence of tryptophan, collagen/pyridinolin/riboflavin, NADH, vitamin A, and vitamin E, respectively. The two first fluorophores were chosen because they are clearly liposoluble while the other fluorophores were chosen because their fluorescence properties can be influenced by their environment (Mazerolles, Devaux, Dufour, Qannari, \& Courcoux, 2002). SFS and FFFS were recorded in duplicates for each sample giving a total of 792 spectra recorded.

\subsection{Data pre-processing}

The proper choice of pre-processing is difficult to assess prior to model validation but pre-processing should maintain or decrease the effective model complexity. So, in order to improve the calibration models different pre-processing methods were investigated. Firstly, noise of the spectral datasets was reduced by using the SavitzkyGolay method with a polynomial order and a filter width of 2 and 12 respectively. Secondly, the scattering effects were minimized by using Standard Normal Variate (SNV), Multiple Scattering Correction (MSC) or by area normalization (reducing the area under each spectrum to a value of 1: AREA). These pre-processing techniques are desired to reduce the (physical) variability between samples due to scatter. Data preprocessing were performed by using the PLS-Toolbox v.7.5 (Eigenvector Research) for MATLAB R2013b.

\subsection{Partial least square regression}

Partial Least Square Regression (PLS-R) models were validated by independent datasets. The initial data sets were divided into two sets, $70 \%$ of the samples were used for the calibration and, $30 \%$ for the validation by using the Nearest Neighbor Thinning method proposed in the PLSToolbox v.7.5 (Eigenvector Research) that permits to select validation samples which best fill out all covariance space. The models performance can be evaluated by different factors. Williams (2003) defines 7 levels of model accuracy based on the $R^{2}$ values obtained for prediction. Nonetheless, considering the $R^{2}$ can conduct to over estimation of the model accuracy. In order to have a clear idea of model performance other statistics such as the Ratio of Performance to Deviation (RPD $=$ SD/RMSEP) factor was considered. Five levels of model accuracy based on the RPD values obtained for prediction were considered (Table 1).

PLS-R analyses were performed by using the PLS-Toolbox v.7.5 (Eigenvector Research) for MATLAB R2013b. 
Table 1

Interpretation of RPD (Ratio of Performance to Deviation) value (Williams, 2003).

\begin{tabular}{lll}
\hline RPD value & Classification & Application \\
\hline $0.00-1.50$ & Not recommended & No application \\
1.50 and 2.00 & Distinguish between high and low values & Rough screening \\
2.00 and 2.50 & Approximate quantitative prediction & Quality control \\
2.50 and 3.00 & Good & Process control \\
$>3.0$ & Excellent & Any application \\
\hline
\end{tabular}

\section{Results}

\subsection{Fatty acid composition measured using the GLC reference method}

The means, ranges, SD (standard deviation) and CV (coefficient of variation) of LT intramuscular lipid and FA concentrations are presented in Table 2. Mean SFA and MUFA were the most abundant FA groups in LT muscle and their contents were similar $(600.05 \mathrm{mg} / 100 \mathrm{~g}$ and $571.25 \mathrm{mg} / 100 \mathrm{~g}$ respectively). On the other hand, mean PUFA contents were 3 times lower (202.95 mg/100 g). Palmitic (16:0) and oleic (18:1 $\Delta 9$ cis) acids were on average the most abundant individual FAs $(299.68 \mathrm{mg} / 100 \mathrm{~g}$ of fresh muscle, ranging from

Table 2

Total lipid and total fatty acid ( $\mathrm{g} / 100 \mathrm{~g}$ fresh meat) contents and fatty acid composition ( $\mathrm{mg} / 100 \mathrm{~g}$ fresh meat) of LT muscle samples.

\begin{tabular}{|c|c|c|c|c|c|}
\hline Component & Mean & Min & Max & SD & $\mathrm{CV}$ \\
\hline Total lipids & 2.07 & 0.78 & 6.06 & 1.22 & $59 \%$ \\
\hline Total FA & 1.39 & 0.36 & 4.35 & 0.98 & $71 \%$ \\
\hline Total FA/total lipids & 0.63 & 0.38 & 0.90 & 0.11 & $17 \%$ \\
\hline \multicolumn{6}{|l|}{ Fatty acid families } \\
\hline Total SFA & 600.05 & 106.53 & 2121.08 & 471.60 & $79 \%$ \\
\hline Total linear SFA & 578.06 & 100.15 & 2067.75 & 457.83 & $79 \%$ \\
\hline Total non-linear SFA & 21.99 & 6.37 & 58.48 & 14.18 & $64 \%$ \\
\hline Total MUFA & 571.25 & 96.20 & 1975.96 & 466.32 & $82 \%$ \\
\hline Total cis MUFA & 483.09 & 77.27 & 1796.27 & 406.62 & $84 \%$ \\
\hline Total trans MUFA & 88.16 & 15.33 & 326.59 & 70.39 & $80 \%$ \\
\hline Total PUFA & 202.95 & 128.38 & 358.24 & 49.27 & $24 \%$ \\
\hline Total n-3 PUFA & 35.20 & 17.88 & 65.45 & 9.94 & $28 \%$ \\
\hline Total n-3 LC PUFA & 14.60 & 7.41 & 26.31 & 3.53 & $24 \%$ \\
\hline Total n-6 PUFA & 159.95 & 99.86 & 274.96 & 37.91 & $24 \%$ \\
\hline Total n-6 cis PUFA & 135.01 & 80.38 & 230.58 & 28.41 & $21 \%$ \\
\hline Total $n-6$ trans PUFA & 24.94 & 2.89 & 94.58 & 21.00 & $84 \%$ \\
\hline Total n-6 LC PUFA & 30.74 & 17.09 & 57.62 & 8.05 & $26 \%$ \\
\hline Total CLA & 6.64 & 0.51 & 23.03 & 5.84 & $88 \%$ \\
\hline \multicolumn{6}{|l|}{ Individual fatty acids } \\
\hline $14: 0$ & 30.26 & 2.38 & 114.69 & 29.88 & $99 \%$ \\
\hline $16: 0$ & 299.68 & 50.64 & 1215.99 & 256.94 & $86 \%$ \\
\hline $18: 0$ & 216.10 & 41.26 & 631.10 & 149.60 & $69 \%$ \\
\hline $18: 1 \Delta 9$ cis & 388.47 & 41.72 & 1512.13 & 343.22 & $88 \%$ \\
\hline $18: 1 \Delta 9$ trans & 5.43 & 0.57 & 22.29 & 4.63 & $85 \%$ \\
\hline $18: 1 \Delta 10-11$ trans & 66.37 & 8.85 & 268.60 & 55.75 & $84 \%$ \\
\hline $18: 2 n-6$ cis cis & 101.84 & 61.97 & 165.82 & 21.25 & $21 \%$ \\
\hline $18: 3 n-3$ & 20.60 & 6.31 & 46.39 & 7.80 & $38 \%$ \\
\hline $20: 3 n-3$ & 0.14 & 0.01 & 1.19 & 0.26 & $186 \%$ \\
\hline $20: 4 n-3$ & 0.80 & 0.01 & 2.75 & 0.62 & $78 \%$ \\
\hline $20: 4 n-6$ & 21.73 & 11.92 & 39.31 & 5.52 & $25 \%$ \\
\hline $20: 5 n-3$ & 3.90 & 2.00 & 6.10 & 1.03 & $26 \%$ \\
\hline $22: 5 n-3$ & 9.26 & 5.15 & 16.47 & 2.23 & $24 \%$ \\
\hline $22: 6 n-3$ & 0.49 & 0.01 & 1.67 & 0.45 & $92 \%$ \\
\hline
\end{tabular}

SFA, saturated FA; Total SFA: linear + non-linear SFA; Linear SFA: 12:0 up to 24:0; Non-linear SFA: iso [14 up to 18] + anteiso [15 + 17], FA mainly synthesized by rumen microorganisms; MUFA, monounsaturated FA; Total MUFA: cis + trans MUFA; Total cis MUFA: 14:1 $\Delta 9$ cis + $15: 1 \Delta 9$ cis $+16: 1 \Delta 9$ cis $+17: 1 \Delta 8$ and $\Delta 9$ cis $+18: 1 \Delta 6$ cis up to $\Delta 15 c i s+20: 1 \Delta 9 c i s+$ 22:1 $\Delta$ 9cis; Total trans MUFA: $16: 1 \Delta 9$ trans $+18: 1 \Delta 6$ trans up to $\Delta 16$ trans; PUFA, polyunsaturated FA; Total PUFA: n-6 PUFA + n-3PUFA + CLA; Total n-3 PUFA: 18:3 n-3 + 20:3 n-3 + $20: 4 n-3+20: 5 n-3+22: 3 n-3+22: 4 n-3+22: 5 n-3+22: 6 n-3$; Total n-6 PUFA: 18:2 $\mathrm{n}-6+18: 3 \mathrm{n}-6+20: 2 \mathrm{n}-6+20: 3 \mathrm{n}-6+20: 4 \mathrm{n}-6+22: 2 \mathrm{n}-6+22: 4 \mathrm{n}-6+22: 5 \mathrm{n}-6$; LC, long chain; CLA, conjugated linoleic acid; Total CLA: 9cis,11trans-CLA + 11cis,13trans-CLA + total cis,cis CLA + total trans,trans CLA.

${ }^{a}$ Abbreviations: SD: standard deviation; Min: minimum; Max: maximum.
50.64 to $1215.19 \mathrm{mg} / 100 \mathrm{~g}$ and $388.47 \mathrm{mg} / 100 \mathrm{~g}$ of fresh muscle, ranging from 41.26 to $1512.13 \mathrm{mg} / 100 \mathrm{~g}$, respectively). Stearic acid (18:0) averaged $216.1 \mathrm{mg} / 100 \mathrm{~g}$, ranging from 41.26 to $631.1 \mathrm{mg} / 100 \mathrm{~g}$. Among PUFA, n-6 PUFA were the most represented in LT muscle (159.95 mg/100 g) with linoleic acid (18:2 n-6) as major FA $(101.84 \mathrm{mg} / 100 \mathrm{~g})$. In contrast, $\mathrm{n}-3$ PUFA contents were low ( $35.2 \mathrm{mg} / 100 \mathrm{~g})$, mainly represented by linolenic acid (18:3 n-3, $20.6 \mathrm{mg} / 100 \mathrm{~g}$ ). All total PUFAs categories (except trans PUFA n-6 and total CLA) and individual 20:3 n-3, 20:4 n-3 and 22:6 n-3 had a lower CV (under 40\%) than individual and total SFAs and MUFAs.

\subsection{Description of fluorescence spectra of meat samples}

Average spectra recorded in the two modes (FFFS and SFS) are presented in Figs. 1 and 2. The Fig. 1 presented fluorescence emission spectra obtained at 5 excitation wavelengths 290, 322, 335, 350, and $382 \mathrm{~nm}$.

The fluorescence spectra obtained after excitation at $290 \mathrm{~nm}$ exhibited a peak at $334 \mathrm{~nm}$. Emission fluorescence spectra obtained after excitation at $322 \mathrm{~nm}$ presented peaks at 353, 379, $527 \mathrm{~nm}$ and a large band from 418 to $524 \mathrm{~nm}$ with a maximum at $466 \mathrm{~nm}$. The emission spectra obtained after excitation at $335 \mathrm{~nm}$ presented different bands, a high one at $369 \mathrm{~nm}$, a large one between 420 and $543 \mathrm{~nm}$ with a maximum at $458 \mathrm{~nm}$ and small ones at 378 and $551 \mathrm{~nm}$. Excitation of meat samples at $350 \mathrm{~nm}$ resulted in an emission fluorescence spectrum presenting a broad band between 420 and $543 \mathrm{~nm}$ with a maximum at $466 \mathrm{~nm}$ and two small peaks at 573 and $596 \mathrm{~nm}$. Finally, the fluorescence spectra obtained after excitation at $382 \mathrm{~nm}$ exhibited peaks at 419, 596, $628 \mathrm{~nm}$ and a broad band between 428 and $546 \mathrm{~nm}$ with a maximum at $468 \mathrm{~nm}$.

Fig. 2 presented average front face synchronous spectra recorded at the different $\Delta \lambda(20,40,60,80,100$ and $120 \mathrm{~nm})$. The spectra recorded with an offset of $20 \mathrm{~nm}$ presented one prominent peak at $327 \mathrm{~nm}$ (emission $347 \mathrm{~nm}$ ) and smaller one between 449 and $525 \mathrm{~nm}$ (emission between 469 and $545 \mathrm{~nm}$ ) with a maximum at $491 \mathrm{~nm}(511 \mathrm{~nm})$. This band shifted to lower wavelength 294, and $293 \mathrm{~nm}$ when the spectra are recorded respectively with offsets 80 and $60 \mathrm{~nm}$. The synchronous spectra of $100 \mathrm{~nm}$ offset presents one large band cantered at $279 \mathrm{~nm}$. The offset of $120 \mathrm{~nm}$ permits to obtain spectra with bands at $271 \mathrm{~nm}$ (emission $391 \mathrm{~nm}$ ), 325 (emission $445 \mathrm{~nm}$ ) and $356 \mathrm{~nm}$ (emission $476 \mathrm{~nm}$ ). Here, the band around $290 \mathrm{~nm}$, which was prominent with the previous offsets ( 20 to $80 \mathrm{~nm}$ ), disappears. The band located between 450 and $525 \mathrm{~nm}$ seems to disappear when the offset value increases.

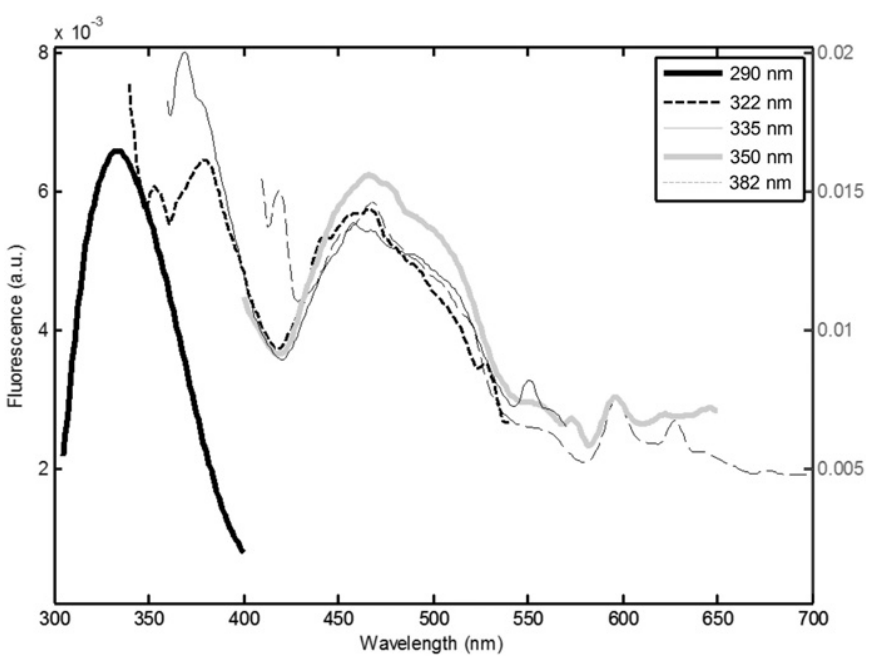

Fig. 1. Average Front Face Fluorescence emission spectra recorded after excitation at 290 $322,335,350$, and $382 \mathrm{~nm}$ on meat samples (a.u. arbitrary unit). 


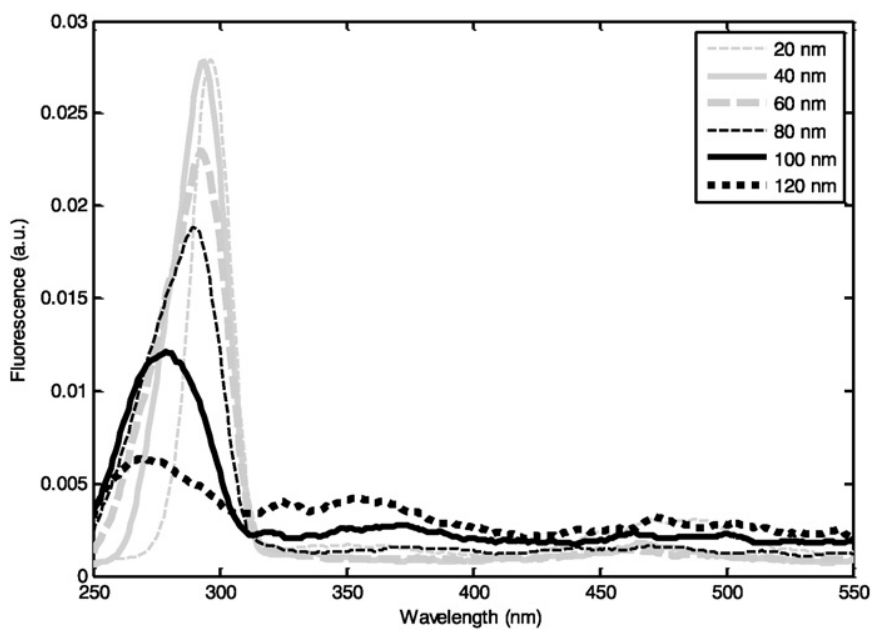

Fig. 2. Average Synchronous Fluorescence spectra recorded at $\Delta \lambda=60,80$ and $120 \mathrm{~nm}$ on meat samples (a.u. arbitrary unit).

\subsection{Prediction of lipid contents with FFFS}

The PLS regression statistics corresponding to the selected equations of the best models developed from FFFS are summarized in Table 3.
With FFFS, the best predictive models were obtained for total lipid content $(\mathrm{RPD}=2.67)$, total FA $(\mathrm{RPD}=2.61)$, SFA ( $\mathrm{RPD}=2.65)$, linear SFA ( RPD $=2.65)$, MUFA ( RPD $=2.62)$, cis MUFA (RPD = 2.77) and for individual unsaturated and mono-unsaturated FA, C14:0 ( RPD $=2.88)$, $\mathrm{C} 16: 0(\mathrm{RPD}=2.61), \mathrm{C} 18: 0(\mathrm{RPD}=2.50), \Delta 9$ cis $\mathrm{C} 18: 1(\mathrm{RPD}=2.80)$, and $\Delta 9$ trans $\mathrm{C} 18: 1(\mathrm{RPD}=2.51)$. All these models were obtained with the $322 \mathrm{~nm}$ excitation band after applying the normalization preprocessing method except for the C14:0 and C16:0 models that were obtained without preprocessing (i.e. RAW).

Seven other models, that can be used to distinguish between high and low values and applied for rough screening (Williams, 2003), were identified for the total trans MUFA ( $\lambda$ ex: $335 \mathrm{~nm}, \mathrm{RPD}=1.54$ ), PUFA ( $\lambda$ ex: 350 nm, RPD = 1.62), n-3 PUFA ( $\lambda$ ex: 322 nm, RPD = 1.54), LC n-3 PUFA ( $\lambda$ ex: 322 nm, RPD $=1.57$ ), n-6 trans PUFA ( $\lambda$ ex: $322 \mathrm{~nm}, \mathrm{RPD}=1.50)$, and CLA ( $\lambda$ ex: $350 \mathrm{~nm}$, RPD = 1.53). These models were calculated after spectral data normalization except for the sum of n-3 PUFA model that was calculated after applying MSC preprocessing to spectra. For the other components, the model accuracies were poor and cannot be used for prediction $(0.89<\mathrm{RPD}<1.49$; $\mathrm{R}_{\mathrm{p}}^{2}<0.35$ ).

\subsection{Prediction of lipid contents with SFS}

The PLS regression statistics of models developed with SFS are summarized in Table 4. Considering SFS, the best predictive models

Table 3

Performance of the best PLS regression models to predict lipids and fatty acid contents of minced beef meat obtained with classical Front Face Fluorescence excitation method ${ }^{\text {a }}$.

\begin{tabular}{|c|c|c|c|c|c|c|c|c|c|}
\hline Component & $\lambda \operatorname{ex}(\mathrm{nm})$ & PPS & LV & $\mathrm{R}_{\mathrm{C}}^{2}$ & $\mathrm{R}_{\mathrm{CV}}^{2}$ & $\mathrm{R}_{\mathrm{P}}^{2}$ & RMSEC & RMSEP & $\mathrm{RPD}_{\mathrm{p}}$ \\
\hline Total lipids & 322 & AREA & 5 & 0.67 & 0.60 & 0.68 & 0.78 & 0.46 & 2.67 \\
\hline Total FA & 322 & AREA & 6 & 0.72 & 0.63 & 0.68 & 0.58 & 0.37 & 2.61 \\
\hline Total FA/total lipids & 322 & AREA & 5 & 0.44 & 0.17 & 0.42 & 0.09 & 0.08 & 1.42 \\
\hline \multicolumn{10}{|l|}{ Fatty acid families } \\
\hline Total SFA & 322 & AREA & 5 & 0.69 & 0.63 & 0.66 & 291.72 & 178.19 & 2.65 \\
\hline Total linear SFA & 322 & AREA & 5 & 0.69 & 0.62 & 0.66 & 283.93 & 172.57 & 2.65 \\
\hline Total non-linear SFA & 322 & AREA & 8 & 0.75 & 0.61 & 0.68 & 7.76 & 6.04 & 2.35 \\
\hline Total MUFA & 322 & AREA & 6 & 0.71 & 0.61 & 0.68 & 279.80 & 177.76 & 2.62 \\
\hline Total cis MUFA & 322 & AREA & 5 & 0.69 & 0.61 & 0.67 & 256.07 & 146.68 & 2.77 \\
\hline Total trans MUFA & 335 & AREA & 9 & 0.65 & 0.43 & 0.51 & 41.78 & 46.07 & 1.54 \\
\hline Total PUFA & 350 & AREA & 7 & 0.40 & 0.06 & 0.45 & 40.33 & 32.37 & 1.62 \\
\hline Total n-3 PUFA & 322 & MSC & 2 & 0.13 & 0.04 & 0.23 & 9.90 & 6.95 & 1.54 \\
\hline Total n-3 LC PUFA & 322 & AREA & 3 & 0.14 & 0.01 & 0.18 & 3.41 & 2.34 & 1.57 \\
\hline Total n-6 PUFA & 350 & MSC & 7 & 0.47 & 0.07 & 0.44 & 29.04 & 24.61 & 1.63 \\
\hline Total n-6 cis PUFA & 335 & SNV & 1 & 0.00 & 0.01 & 0.06 & 31.44 & 24.21 & 1.22 \\
\hline Total n-6 trans PUFA & 322 & AREA & 6 & 0.46 & 0.34 & 0.33 & 16.22 & 14.80 & 1.50 \\
\hline Total n-6 LC PUFA & 335 & SNV & 5 & 0.05 & 0.05 & 0.14 & 8.27 & 6.21 & 1.37 \\
\hline Total CLA & 350 & AREA & 10 & 0.79 & 0.44 & 0.58 & 2.75 & 3.90 & 1.53 \\
\hline \multicolumn{10}{|l|}{ Individual fatty acids } \\
\hline $14: 0$ & 322 & RAW & 7 & 0.77 & 0.66 & 0.75 & 15.27 & 11.23 & 2.88 \\
\hline $16: 0$ & 322 & RAW & 10 & 0.83 & 0.71 & 0.65 & 119.98 & 98.54 & 2.61 \\
\hline $18: 0$ & 322 & AREA & 5 & 0.73 & 0.67 & 0.66 & 85.50 & 59.91 & 2.50 \\
\hline $18: 1 \Delta 9$ cis & 322 & AREA & 5 & 0.69 & 0.61 & 0.68 & 214.88 & 122.79 & 2.80 \\
\hline $18: 1 \Delta 9$ trans & 322 & AREA & 6 & 0.64 & 0.50 & 0.63 & 3.12 & 1.85 & 2.51 \\
\hline $18: 1 \Delta 10-11$ trans & 335 & AREA & 9 & 0.60 & 0.35 & 0.48 & 34.813 & 37.244 & 1.49 \\
\hline $18: 2$ n-6 cis cis & 350 & SNV & 1 & 0.01 & 0.06 & 0.09 & 23.34 & 18.11 & 1.26 \\
\hline $18: 3 n-3$ & 322 & MSC & 2 & 0.13 & 0.05 & 0.17 & 7.67 & 6.05 & 1.37 \\
\hline $20: 3 n-3$ & 290 & MSC & 10 & 0.15 & 0.00 & 0.33 & 0.43 & 0.36 & 0.89 \\
\hline $20: 4 n-3$ & 322 & AREA & 6 & 0.12 & 0.00 & 0.16 & 0.62 & 0.48 & 1.39 \\
\hline $20: 4 n-6$ & 290 & AREA & 3 & 0.05 & 0.00 & 0.00 & 5.65 & 4.02 & 1.45 \\
\hline $20: 5 n-3$ & 322 & MSC & 2 & 0.07 & 0.00 & 0.10 & 1.02 & 0.88 & 1.21 \\
\hline $22: 5 n-3$ & 322 & AREA & 4 & 0.21 & 0.05 & 0.15 & 2.00 & 1.65 & 1.37 \\
\hline $22: 6 n-3$ & 290 & MSC & 3 & 0.09 & 0.04 & 0.28 & 0.43 & 0.37 & 1.24 \\
\hline
\end{tabular}

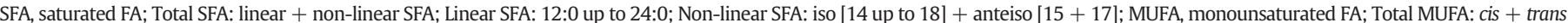

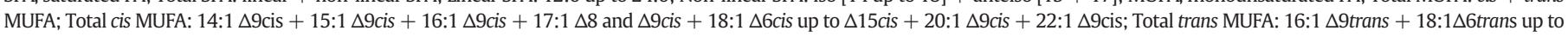

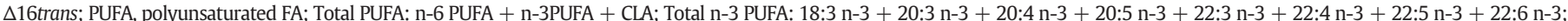

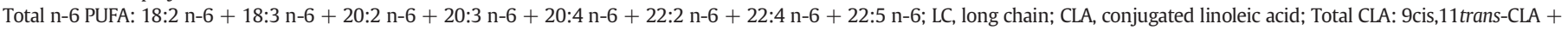
11cis,13trans-CLA + Total cis,cis CLA + Total trans,trans CLA.

a Abbreviations: $\lambda$ ex: excitation wavelength; PPS: preprocess; RAW: without preprocessing; SNV: Standard Normal Variate; AREA: normalization; LV: loading vector; MSC: Multiple

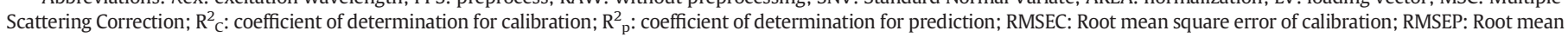
square error of prediction; RPDp: Ratio of Performance to Deviation for prediction. 
were obtained for the total lipid content $(\mathrm{RPD}=2.76)$, MUFA (RPD $=$ 2.55), cis MUFA (RPD = 2.77), C14:0 (RPD = 2.76), $\Delta 9$ cis C18:1, $(\mathrm{RPD}=2.63)$, and $\Delta 9$ trans $\mathrm{C} 18: 1(\mathrm{RPD}=2.68)$. Based on the RPD and $R_{p}^{2}$, those models can be used for process control (Williams, 2003). The different models were calculated with an offset value of $60 \mathrm{~nm}$ (maximum emission $353 \mathrm{~nm}$ ) except for the PLS-R model of $\Delta 9$ trans $\mathrm{C} 18: 1$ obtained with an offset value of $120 \mathrm{~nm}$ (maximum emission $391 \mathrm{~nm}$ ). All these predictive models were obtained with the data corrected by SNV preprocessing except for the predictive model of the C14:0 that was calculated after correcting spectra with the AREA preprocessing method.

The regression models of total amount of FA ( RPD $=2.46)$, SFA $(\mathrm{RPD}=2.40)$, linear SFA ( RPD = 2.39), CLA ( RPD = 2.25), C16:0 $(\mathrm{RPD}=2.29), \mathrm{C} 18: 0$ ( $\mathrm{RPD}=2.39)$ can be used for approximate quantitative prediction. Those models were obtained with an offset of $120 \mathrm{~nm}$ except for total amount of FA and C16:0 calculated with two offsets 60 and $80 \mathrm{~nm}$ respectively. These models were obtained after applying the SNV preprocessing method to spectral data except

Table 4

Performance of the best PLS regression models to predict lipids and fatty acid contents of minced beef obtained with Synchronous Fluorescence excitation method ${ }^{\mathrm{a}}$.

\begin{tabular}{|c|c|c|c|c|c|c|c|c|}
\hline Component & $\Delta \lambda(\mathrm{nm})$ & PPS & LV & $\mathrm{R}^{2} \mathrm{C}$ & $\mathrm{R}_{\mathrm{P}}^{2}$ & RMSEC & RMSEP & $\mathrm{RPD}_{\mathrm{p}}$ \\
\hline Total lipids & 60 & AREA & 8 & 0.87 & 0.77 & 0.46 & 0.46 & 2.76 \\
\hline Total FA & 60 & AREA & 8 & 0.87 & 0.80 & 0.35 & 0.40 & 2.46 \\
\hline Total FA/total lipids & 80 & AREA & 4 & 0.26 & 0.58 & 0.10 & 0.08 & 1.47 \\
\hline \multicolumn{9}{|l|}{ Fatty acid families } \\
\hline Total SFA & 120 & SNV & 7 & 0.93 & 0.80 & 122.32 & 195.24 & 2.40 \\
\hline Total linear SFA & 120 & SNV & 7 & 0.93 & 0.80 & 118.82 & 189.68 & 2.39 \\
\hline Total non-linear SFA & 60 & AREA & 7 & 0.84 & 0.76 & 5.80 & 6.09 & 2.37 \\
\hline Total MUFA & 60 & SNV & 7 & 0.86 & 0.79 & 175.83 & 182.27 & 2.55 \\
\hline Total cis MUFA & 60 & SNV & 7 & 0.87 & 0.81 & 145.76 & 153.48 & 2.62 \\
\hline Total trans MUFA & 60 & AREA & 5 & 0.52 & 0.54 & 51.39 & 38.17 & 1.95 \\
\hline Total PUFA & 120 & AREA & 4 & 0.48 & 0.48 & 36.93 & 29.36 & 1.76 \\
\hline Total n-3 PUFA & 40 & MSC & 6 & 0.46 & 0.37 & 7.80 & 5.78 & 1.85 \\
\hline Total n-3 LC PUFA & 60 & SNV & 3 & 0.25 & 0.03 & 3.29 & 2.88 & 1.32 \\
\hline Total n-6 PUFA & 120 & AREA & 4 & 0.44 & 0.39 & 29.12 & 26.45 & 1.47 \\
\hline Total n-6 cis PUFA & 20 & MSC & 5 & 0.17 & 0.13 & 25.33 & 24.78 & 1.17 \\
\hline Total n- 6 trans PUFA & 60 & AREA & 6 & 0.56 & 0.43 & 15.08 & 12.51 & 1.82 \\
\hline Total n-6 LC PUFA & 40 & AREA & 10 & 0.25 & 0.16 & 6.65 & 8.02 & 1.07 \\
\hline Total CLA & 120 & AREA & 5 & 0.72 & 0.71 & 3.01 & 2.52 & 2.25 \\
\hline \multicolumn{9}{|l|}{ Individual fatty acids } \\
\hline $14: 0$ & 60 & AREA & 8 & 0.86 & 0.83 & 11.38 & 10.95 & 2.76 \\
\hline $16: 0$ & 80 & SNV & 8 & 0.92 & 0.77 & 73.36 & 115.14 & 2.29 \\
\hline $18: 0$ & 120 & SNV & 5 & 0.81 & 0.78 & 64.60 & 62.16 & 2.39 \\
\hline $18: 1 \Delta 9$ cis & 60 & SNV & 7 & 0.87 & 0.81 & 121.59 & 128.85 & 2.63 \\
\hline $18: 1 \Delta 9$ trans & 120 & SNV & 4 & 0.70 & 0.74 & 2.37 & 1.63 & 2.68 \\
\hline $18: 1 \Delta 10-11$ trans & 20 & AREA & 7 & 0.69 & 0.50 & 30.75 & 32.46 & 1.71 \\
\hline $18: 2$ n-6 cis cis & 20 & MSC & 5 & 0.16 & 0.09 & 19.33 & 18.50 & 1.20 \\
\hline $18: 3 n-3$ & 40 & AREA & 6 & 0.38 & 0.39 & 6.59 & 4.61 & 1.83 \\
\hline $20: 3 n-3$ & 80 & MSC & 10 & 0.15 & 0.00 & 0.24 & 0.26 & 1.09 \\
\hline $20: 4 n-3$ & 60 & AREA & 2 & 0.08 & 0.11 & 0.66 & 0.42 & 1.64 \\
\hline $20: 4 n-6$ & 100 & AREA & 10 & 0.29 & 0.05 & 4.48 & 5.89 & 1.06 \\
\hline $20: 5 n-3$ & 40 & SNV & 5 & 0.29 & 0.11 & 0.92 & 0.76 & 1.44 \\
\hline $22: 5 n-3$ & 120 & AREA & 4 & 0.33 & 0.16 & 1.86 & 1.76 & 1.30 \\
\hline $22: 6 n-3$ & 120 & RAW & 6 & 0.51 & 0.34 & 0.32 & 0.32 & 1.41 \\
\hline
\end{tabular}

SFA, saturated FA; Total SFA: linear + non-linear SFA; Linear SFA: 12:0 up to 24:0; Non-linear SFA: iso [14 up to 18] + anteiso [15 + 17]; MUFA, monounsaturated FA; Total MUFA: cis + trans MUFA; Total cis MUFA: $14: 1 \Delta$ cis $+15: 1 \Delta$ cis $+16: 1 \Delta 9$ cis $+17: 1 \Delta 8$ and $\Delta 9$ cis + 18:1 $\Delta 6$ cis up to $\Delta 15$ cis $+20: 1 \Delta 9$ cis $+22: 1 \Delta$ cis; Total trans MUFA: $16: 1 \Delta 9$ trans + 18:1 $\Delta 6$ transup to $\Delta 16$ trans; PUFA, polyunsaturated FA; Total PUFA: n-6 PUFA $+\mathrm{n}$ 3PUFA + CLA; Total n-3 PUFA: 18:3 n-3 + 20:3 n-3 + 20:4 n- $3+20: 5 n-3+22: 3$ $\mathrm{n}-3+22: 4 \mathrm{n}-3+22: 5 \mathrm{n}-3+22: 6 \mathrm{n}-3$; Total n-6 PUFA: $18: 2 \mathrm{n}-6+18: 3 \mathrm{n}-6+$ 20:2 n-6 + 20:3 n-6 + 20:4 n-6 + 22:2 n-6 + 22:4 n-6 + 22:5 n-6; LC, long chain; CLA, conjugated linoleic acid; Total CLA: 9cis,11trans-CLA + 11cis,13trans-CLA + Total cis,cis CLA + Total trans,trans CLA.

a Abbreviations: $\Delta \lambda$ : offset in nm; PPS: preprocess; RAW: without preprocessing; SNV: Standard Normal Variate; AREA: normalization; LV: loading vector; MSC: Multiple Scattering Correction; $\mathrm{R}_{\mathrm{C}}^{2}$ : coefficient of determination for calibration; $\mathrm{R}_{\mathrm{p}}{ }_{\mathrm{p}}$ : coefficient of determination for prediction; RMSEC: root mean square error of calibration; RMSEP: root mean square error of prediction; RPDp: residual predictive deviation for prediction. for total FA and CLA that were calculated after performing the normalization preprocessing method.

Seven other models, that can be used to distinguish between high and low values and applied for rough screening (Williams, 2003), were identified for the sum of MUFA trans (RPD $=1.95$ ), PUFA $(\mathrm{RPD}=1.76), \mathrm{n}-3$ PUFA (RPD = 1.85), $\mathrm{n}-6$ trans PUFA $(\mathrm{RPD}=1.82)$, $\Delta 10-11$ trans $\mathrm{C} 18: 1(\mathrm{RPD}=1.71), \mathrm{n}-3 \mathrm{C} 18: 3(\mathrm{RPD}=1.83)$, and $\mathrm{n}-3$ $\mathrm{C} 20: 4(\mathrm{RPD}=1.64)$. These different predictive models were calculated with the normalization preprocessing method except for the sum of n-3 PUFA predictive model that was obtained after applying the MSC method to spectral data.

The accuracy of the other predictive models was low based on their RPD values $\left(1.06<\mathrm{RPD}<1.47\right.$ and $\left.\mathrm{R}_{\mathrm{p}}^{2}<0.58\right)$.

\section{Discussion}

\subsection{Total lipids and fatty acids prediction in meat}

A few studies have attempted to confirm the ability of fluorescence spectroscopy to predict total lipids of meat (Brondum et al., 2000; Wold, Kvaal et al., 1999; Wold, Lundby et al., 1999). Wold, Lundby et al. (1999) reported a $R^{2}$ of 0.71 and a RPD of 1.86 for intact beef Longissimus dorsi muscle and Brondum et al. (2000) found lower $\mathrm{R}^{2}$ (0.57) and RPD (1.52) on pork intact muscles. The higher predictive accuracy of the models reported in the present work (FFFS: $\mathrm{R}^{2}=0.68$ and $\mathrm{RPD}=2.61$; $\mathrm{SFS}: \mathrm{R}^{2}=0.80, \mathrm{RPD}=2.46$ ) are probably associated with two factors: (i) a higher variability in the data base: increasing variability by the use of several breeds fed, several diets, and muscle type in sampling had a positive effect on beef FA prediction (Brondum et al., 2000; Mourot et al., 2015); (ii) the preparation of the samples: in this study, meat samples were transformed into fine powder under liquid nitrogen whereas in other studies, samples were intact. The fluorescence measurement can be considered as a surface measurement and the grinding of the sample can permit to record a fluorescence signal that is more representative of the sample. NIR spectroscopy is undoubtedly the most used method for predicting total lipids in beef, some studies after cross-validation reported $a \mathrm{R}^{2}{ }_{\mathrm{cv}}$ and RPD higher than 0.9 and 3.5 respectively (De Marchi, Berzaghi, Boukha,Mirisola, \& Gallo, 2007; Mourot et al., 2015; Tøgersen, Arnesen, Nilsen, \& Hildrum, 2003) suggesting that this spectral method presented probably a higher accuracy to predict total lipids in beef.

As far as we know, this study brings new insight regarding the ability of FFFS to predict FA composition of beef because no study reported equivalent investigation. According to Williams (2003), the regression models calculated for total SFA, linear SFA, MUFA, cis MUFA and for some individual unsaturated and mono-unsaturated FA (C14:0, C16:0, C18:0, $\Delta 9$ cis C18:1, and $\Delta 9$ trans C18:1) could be used for process control $\left(R_{p}^{2} \geq 0.63\right.$ and $\left.\operatorname{RPD}_{\mathrm{p}} \geq 2.50\right)$ and the one obtained for nonlinear SFA would be suited for approximate quantitative prediction.

The poor predictive models calculated for the other FAs (i.e. total or individual MUFA and PUFA) except the oleic acid $\Delta 9$ cis C18:1, could be attributed either to a narrow variation range of the reference values (giving small CV). Nonetheless, this hypothesis was not verified for total CLA, total trans MUFA, 18:1 $\Delta$ 10-11 trans, 20:3 n-3, 20:4 n-3, and 22:6 n-3 that presented high CV and low RPD values. We suggested that those components are not well predicted due both to a large RMSEP compared to SD of the reference values (Prevolnik, Skrlep, Skorjanc, \& Candek-Potokar, 2010) and to their lower concentration in meat (Tables 2 and 3). Indeed, the concentration of MUFA and PUFA are lower compared to the other FA except for the oleic acid. Moreover, we assumed the presence of a different minimum threshold of detection between the SFA and MUFA. Indeed, the total MUFA and cis MUFA are correctly predicted compared to individual MUFA except for oleic acid which is the most abundant FA.

The best predictive models discussed above were obtained by using three excitation wavelengths (322, 335 or $350 \mathrm{~nm}$ ) that were previously 
reported as being sensitive to meat fat. The $322 \mathrm{~nm}$ was assigned to vitamin A (Skjervold et al., 2003). The $335 \mathrm{~nm}$ excitation wavelength was used to predict intramuscular fat content on beef (Wold, Kvaal et al., 1999; Wold, Lundby et al., 1999). Nonetheless, this excitation band was also reported as sensitive to NADH (Karoui, Thomas, \& Dufour, 2006). Even if its concentration is small in meat sample (Chan et al., 1996; Gatellier, Hamelin, Durand, \& Renerre, 2001), we considered the $350 \mathrm{~nm}$ excitation wavelength associated to the fluorescence of vitamin E. Nonetheless, some inconsistencies concerning the assignment of this band to vitamin $\mathrm{E}$ have to be pointed out in such a complex product (Sikorska, Khmelinskii, \& Sikorski, 2011).

No interesting models were obtained with the 290 and $382 \mathrm{~nm}$ excitation bands probably due to their low relation with meat fat. Indeed, the $290 \mathrm{~nm}$ band was mainly assigned to tryptophan residues in proteins and usually applied as a fingerprint to discriminate muscles (Dufour \& Frencia, 2001; Sahar, Boubellouta, Portanguen, Kondjoyan, \& Dufour, 2009), to predict microbial spoilage of meat (Aït-Kaddour et al., 2011) and chicken breast filet (Sahar, Boubellouta, \& Dufour, 2011). For the $382 \mathrm{~nm}$ band, Wold, Kvaal et al. (1999), Egelandsdal, Wold, Sponnich, Neegård, and Hildrum (2002) and Skjervold et al. (2003) have shown that this excitation wavelength is more adapted to extract information related to connective tissue. This was confirmed by Sahar et al. (2009), nonetheless they reported that riboflavin could also be excited.

The SFS mode used in this study demonstrates that spectra recorded on meat samples exhibit peaks corresponding to different fluorophores as reported by Sahar et al. (2009). The results demonstrated that the influence of $\Delta \lambda$ can be substantial on the shape, location, and signal intensity of the fluorescence peaks. Indeed, depending of the $\Delta \lambda$ value, synchronous fluorescence spectra are substantially modified. As previously noted (Boubellouta \& Dufour, 2008), SFS makes it possible to narrow the spectral bands and, compared to emission or excitation spectra, to have information on the fluorescence properties of several intrinsic fluorophores on a given spectrum. This suggests the potential of synchronous fluorescence spectra to be suited for the prediction of different lipid components in beef.

As far as we know, this study brings also new insight regarding the ability of SFS to predict total FA and other lipids of beef because no study reported equivalent investigation. As discussed for FFFS, the regression models calculated for total lipids, MUFA, cis MUFA and individual unsaturated and mono-unsaturated FA (C14:0, C18:1, and $\Delta 9$ trans $\mathrm{C} 18: 1)$ could be used for process control and the one obtained for total FA, linear SFA, C16:0, and C18:0 would be suited for approximate quantitative prediction (Williams, 2003). The lower accuracy of predictive models calculated for the other FAs can be attributed, as for FFFS, either to a narrow variation range of the reference values, to large RMSEP compared to SD of the reference values and to their lower concentration in meat. Moreover, a higher ability of SFS to predict SFA can be noted compared to FFFS.

In the present study, three offsets can be identified as the most suited for meat lipid prediction, 60,80 , and $120 \mathrm{~nm}$. The synchronous fluorescence excitation spectra obtained with those three offsets presented different peaks that can be related to specific fluorophores. The spectral band identified around $296 \mathrm{~nm}$ seems to originate from the fluorescence of the protein tryptophan residues (Boubellouta \& Dufour, 2008; Sahar et al., 2009). While, the assignment of the band, between 450 and $525 \mathrm{~nm}$, to a specific fluorophore remains unclear. For example, it was previously assigned to riboflavin and to Maillard reaction products (Sahar et al., 2009; Yaacoub et al., 2009).

As previously mentioned, no studies have investigated the potential of fluorescence spectroscopy to predict individual or total FA composition of ground LT muscles. Nonetheless, few studies have used NIR spectroscopy to predict those components in ground LT muscles (Cecchinato et al., 2012; Mourot et al., 2015; Sierra et al., 2008). The models reported in those studies for 14:0, 16:0, 18:0, 18:1 $\Delta 9,20: 3$ $n-3,22: 6 n-3$ were more accurate than those reported in the present study. Nonetheless, the models for 18:1 $\Delta 9$ trans, 20:4 n-6, 20:5 n-3, and 22:5 n-3 presented higher RPD values whatever the fluorescence spectroscopy method (SFS or FFFS) compared to the NIR spectroscopy ( 0.40 in average).

For total SFA, linear SFS, MUFA, cis MUFA higher predictive statistics were reported for NIR spectroscopy. In the study of Mourot et al. (2015), that reported the best statistics for predictive models, the RPD $\mathrm{cv}_{\mathrm{cv}}$ was improved by an average value of 0.98 . Total PUFA, n-3 PUFA, n-3 LC PUFA, n-6 PUFA, n-6 trans PUFA, n-6 LC PUFA were more accurately predicted by fluorescence spectroscopy (SFF and FFFS) compared to NIR spectroscopy (the RPD is improved in our study by a value of 0.32 ). For total trans MUFA, total CLA, 22:6n-3, and 18:3 n-3 higher RPD value $(+0.12$ in average) was obtained only by SFS excitation method compared to NIR spectroscopy. Those observations could suggest that fluorescence spectroscopy is more suited for predicting the PUFA than NIR spectroscopy. Nonetheless, it seems whatever the method used that the major FAs (representing over $1 \%$ of the total FAs identified such as 14:0, 16:0, 18:0, 18:1 $\Delta 9, \ldots$ ) were more accurately predicted than minor FAs (Mourot et al., 2015). Also, total and many individual SFAs and MUFAs were better predicted than total and individual PUFAs.

The best regression models for total fat, lipids and each FAs were obtained using different mathematical preprocessing. In the present study, nearly all-preprocessed models are simpler or more parsimonious (i.e. use fewer PLS factors) than the non-preprocessed models (i.e. RAW models), independent of the fluorescence spectra used, FFFS or SFS. The best preprocessing method differs between components considered suggesting that it is impossible to know beforehand which preprocessing would lead to the most accurate model previously reported (Fernández-Cabanás, Garrido-Varo, Olmo, Pedro, \& Dardenne, 2007). Nonetheless, it is interesting to note both that the models created from normalized (i.e. AREA) spectra are generally the simplest models and the best preprocessing method. This is more obvious for the models calculated with FFFS that gave 20 components among 33 compared to the models calculated with SFS that presented 16 components among 33 presenting the normalization as the best preprocessing method (Tables 3 and 4). Those results suggested that the normalization approach appears as a good preprocessing strategy for the prediction of fat and fatty acid contents from fluorescence spectra. This study shows, and recalled, that a normalization is not at all a neutral operation and has to be carefully chosen.

\subsection{Comparison of the ability of FFFS and SFS to predict fatty acid composition}

The comparison between the two fluorescence excitation modes was conducted whenever a predictive model was usable for process control (RPD $\geq 2.50$ ). Compared to the SFS, the models obtained with FFFS presented generally lower values of $\mathrm{R}_{\mathrm{p}}^{2}$, loading vectors, RMSEP and higher values of RPD factors. The b-coefficient obtained with the SFS presented a noisy shape (results not shown) indicating an over fitting of models calculated. This implies that the models obtained with FFFS are generally more accurate and simplest or more parsimonious than the models obtained with SFS data. Considering that meat is a complex and anisotropic matrix presenting different physicochemical properties, the use of only some excitation or emission wavelengths for the excitation of its intrinsic fluorophores would have limited the ability of the FFFS to predict efficiently some lipids in meat compared to the SFS excitation mode. Nonetheless, the present study clearly shows that the FFFS is the best method for prediction of fat and fatty acid contents in meat.

Those results can be assigned to the fact that a synchronous fluorescence spectrum exhibits peaks corresponding to several different fluorophores. These compounds are present at the sample surface resulting in an increase of spectral loss and interferences. This induces instability in models compared to the FFFS that allows exciting a 
fluorophore more specifically. This observation is in agreement with the observation of Patra and Mishra (2002) who reported that in spite of the sensitivity, selectivity, for multi-component analysis, SFS fails for very complex mixtures.

\section{Conclusion}

This study can be considered as a first step to demonstrate the ability of fluorescence spectroscopy to predict FA contents of the LT muscle coming from three breeds of cattles. This method globally appears as a promising tool to support the current effort of research carried out to improve the nutritional quality of meat products. The results suggested that the fluorescence spectroscopy is more suited to measure SFA and MUFA because this method failed when the prediction of PUFA was investigated. It seems that a higher minimum threshold was necessary to predict correctly the PUFA compared to the SFA. Indeed, the total MUFA and cis MUFA are correctly predicted compared to individual MUFA except for oleic acid which is the most abundant FA. However more effort should be done to validate those hypotheses. Taking into account the number of samples used in the present study and experimental conditions, the models obtained are not ready to be used in practical conditions. A more complete calibration and validation phases, with different muscle categories, feeding regimes and higher number of breeds will be necessary to build robust predictive models and to ensure that fluorescence spectroscopy is adapted for prediction of meat fat composition and for industrial applications.

\section{References}

Aït-Kaddour, A., Boubellouta, T., \& Chevallier, I. (2011). Development of a portable spectrofluorimeter for measuring the microbial spoilage of minced beef. Meat Science, $88(4), 675-681$.

Bauchart, D., Gladine, C., Gruffat, D., Leloutre, L., \& Durand, D. (2005). Effects of diets supplemented with oil seeds and vitamin $\mathrm{E}$ on specific fatty acids of rectus abdominis muscle in Charolais fattening bulls. Indicators of Milk and Beef Quality, 112, 431-436.

Benjamin, S., \& Spener, F. (2009). Conjugated linoleic acids as functional food: An insight into their health benefits. Nutrition and Metabolism, 6(36), 1-13.

Boubellouta, T., \& Dufour, E. (2008). Effects of mild heating and acidification on the molecular structure of milk components as investigated by synchronous front-face fluorescence spectroscopy coupled with parallel factor analysis. Applied Spectroscopy, 62(5), 490-496.

Brondum, J., Munck, L., Henckel, P., Karlsson, A., Tornberg, E., \& Engelsen, S.B. (2000). Prediction of water-holding capacity and composition of porcine meat by comparative spectroscopy. Meat Science, 55(2), 177-185.

Cecchinato, A., De Marchi, M., Penasa, M., Casellas, J., Schiavon, S., \& Bittante, G. (2012). Genetic analysis of beef fatty acid composition predicted by near-infrared spectroscopy. Journal of Animal Science, 60(2), 429-438.

Chan, W. K. M., Hakkarainen, K., Faustman, C., Schaefer, D. M., Scheller, K. K., \& Liu, Q. (1996). Dietary vitamin E effect on color stability and sensory assessment of spoilage in three beef muscles. Meat Science, 42(4), 387-399.

Chardigny, J. M., Destaillats, F., Malpuech-Brugère, C., Moulin, J., Bauman, D. E., Lock, A. L., ... Sébédio, J. L. (2008). Do trans fatty acids from industrially produced sources and from natural sources have the same effect on cardiovascular disease risk factors in healthy subjects? Results of the trans fatty acids collaboration (TRANSFACT) study. The American Journal of Clinical Nutrition, 87(3), 558-566.

De Marchi, M., Berzaghi, P., Boukha, A., Mirisola, M., \& Gallo, L. (2007). Use of near infrared spectroscopy for assessment of beef quality traits. Italian Journal of Animal Science, $6(1 \mathrm{~s}), 421-423$.

Dufour, E., \& Frencia, J. P. (2001). Les spectres de fluorescence frontale: une empreinte digitale de la viande. Viandes et Produits Carnés, 22(1), 9-14.

Egelandsdal, B., Dingstad, G., Tøgersen, G., Lundby, F., \& Langsrud, O. (2005). Autofluorescence quantifies collagen in sausage batters with a large variation in myoglobin content. Meat Science, 69(1), 35-46.

Egelandsdal, B., Wold, J. P., Sponnich, A., Neegård, S., \& Hildrum, K. I. (2002). On attempts to measure the tenderness of Longissimus Dorsi muscles using fluorescence emission spectra. Meat Science, 60(2), 187-202.

Fernández-Cabanás, V. M., Garrido-Varo, A., Olmo, J. G., Pedro, E. D., \& Dardenne, P. (2007). Optimisation of the spectral pre-treatments used for Iberian pig fat NIR calibrations. Chemometrics and Intelligent Laboratory Systems, 87(1), 130-138.

Folch, J., Lees, M., \& Sloane, G. H. S. (1957). A simple method for the isolation and purification of total lipids from animal tissues. Journal of Biological Chemistry, 226(1), 497-509.

Gatellier, P., Gomez, S., Gigaud, V., Berri, C., Bihan-Duval, E. L., \& Santé-Lhoutellier, V. (2007). Use of a fluorescence front face technique for measurement of lipid oxidation during refrigerated storage of chicken meat. Meat Science, 76(3), 543-547.

Gatellier, P., Hamelin, C., Durand, Y., \& Renerre, M. (2001). Effect of a dietary vitamin E supplementation on colour stability and lipid oxidation of air and modified atmospherepackaged beef. Meat Science, 59(2), 133-140.
Gatellier, P., Santé-Lhoutellier, V., Portanguen, S., \& Kondjoyan, A. (2009). Use of meat fluorescence emission as a marker of oxidation promoted by cooking. Meat Science, 83(4), 651-656.

Gidding, S. S., Dennison, B. A., Birch, L. L., Daniels, S. R., Gillman, M. W., Gilman, M. W., Van Horn, L. (2005). Dietary recommendations for children and adolescents: A guide for practitioners: Consensus statement from the American heart association. Circulation, 112(13), 2061-2075.

Gruffat, D., Cherfaoui, M., Bonnet, M., Thomas, A., Bauchart, D., \& Durand, D. (2013). Breed and dietary linseed affect gene expression of enzymes and transcription factors involved in n-3 long chain polyunsaturated fatty acids synthesis in longissimus thoracis muscle of bulls. Journal of Animal Science, 91(7), 3059-3069.

Guy, F., Prache, S., Thomas, A., Bauchart, D., \& Andueza, D. (2011). Prediction of lamb meat fatty acid composition using near-infrared reflectance spectroscopy (NIRS). Food Chemistry, 127(3), 1280-1286.

Hooper, L., Abdelhamid, A., Moore, H. J., Douthwaite, W., \& Murray, C. (2012). Effect of reducing total fat intake on body weight: Systematic review and meta-analysis of randomised controlled trials and cohort studies. British Medical Journal, 345(e7666), 1-15.

Karoui, R., \& Blecker, C. (2010). Fluorescence spectroscopy measurement for quality assessment of food systems-A review. Food and Bioprocess Technology, 4(3), 364-386.

Karoui, R., Thomas, E., \& Dufour, E. (2006). Utilisation of a rapid technique based on frontface fluorescence spectroscopy for differentiating between fresh and frozen-thawed fish fillets. Food Research International, 39(3), 349-355.

Lloyd, J. B. (1971). Synchronized excitation of fluorescence emission spectra. Nature Physical Science, 231, 64-65.

Mazerolles, G., Devaux, M. F., Dufour, E., Qannari, E. M., \& Courcoux, P. (2002). Chemometric methods for the coupling of spectroscopic techniques and for the extraction of the relevant information contained in the spectral data tables. Chemometrics and Intelligent Laboratory Systems, 63(1), 57-68.

McAfee, A. J., McSorley, E. M., Cuskelly, G. J., Moss, B. W., Wallace, J. M. W., Bonham, M. P., \& Fearon, A. M. (2010). Red meat consumption: An overview of the risks and benefits. Meat Science, 84(1), 1-13.

Møller, J. K. S., Parolari, G., Gabba, L., Christensen, J., \& Skibsted, L. H. (2003). Monitoring chemical changes of dry-cured Parma ham during processing by surface autofluorescence spectroscopy. Journal of Agricultural and Food Chemistry, 51(5) 1224-1230.

Mourot, B. P., Gruffat, D., Durand, D., Chesneau, G., Mairesse, G., \& Andueza, D. (2015). Breeds and muscle types modulate performance of near-infrared reflectance spectroscopy to predict the fatty acid composition of bovine meat. Meat Science, 99, 104-112.

Olsen, E., Vogt, G., Ekeberg, D., Sandbakk, M., Pettersen, J., \& Nilsson, A. (2005). Analysis of the early stages of lipid oxidation in freeze-stored pork back fat and mechanically recovered poultry meat. Journal of Agricultural and Food Chemistry, 53(2), 338-348.

Patra, D., \& Mishra, A. K. (2002). Recent developments in multi-component synchronous fluorescence scan analysis. Trends in Analytical Chemistry, 21(12), 787-798.

Prevolnik, M., Skrlep, M., Skorjanc, D., \& Candek-Potokar, M. (2010). Application of near infrared spectroscopy to predict chemical composition of meat and meat products. Technologija Mesa, 51(2), 133-142.

Prieto, N., López-Campos, Ó., Aalhus, J. L., Dugan, M. E. R., Juárez, M., \& Uttaro, B. (2014). Use of near infrared spectroscopy for estimating meat chemical composition, quality traits and fatty acid content from cattle fed sunflower or flaxseed. Meat Science, 98(2), 279-288.

Riccardi, G., Giacco, R., \& Rivellese, A. A. (2004). Dietary fat, insulin sensitivity and the metabolic syndrome. Clinical Nutrition, 23(4), 447-456.

Sahar, A., Boubellouta, T., \& Dufour, É. (2011). Synchronous front-face fluorescence spectroscopy as a promising tool for the rapid determination of spoilage bacteria on chicken breast fillet. Food Research International, 44(1), 471-480.

Sahar, A., Boubellouta, T., Portanguen, S., Kondjoyan, A., \& Dufour, É. (2009). Synchronous front-face fluorescence spectroscopy coupled with parallel factors (PARAFAC) analysis to study the effects of cooking time on meat. Journal of Food Science, 74(9), 534-539.

Sahar, A., Portanguen, S., Kondjoyan, A., \& Dufour, E. (2010). Potential of synchronous fluorescence spectroscopy coupled with chemometrics to determine the heterocyclic aromatic amines in grilled meat. European Food Research and Technology, 231(5), 803-812.

Scislowski, V., Durand, D., Gruffat, D., \& Bauchart, D. (2004). Dietary linoleic acid-induced hypercholesterolemia and accumulation of very light HDL in steers. Lipids, 39(2), 125-133.

Scollan, N. D., Dannenberger, D., Nuernberg, K., Richardson, I., MacKintosh, S., Hocquette, J F., \& Moloney, A. P. (2014). Enhancing the nutritional and health value of beef lipids and their relationship with meat quality. Meat Science, 97(3), 384-394.

Sierra, V., Aldai, N., Castro, P., Osoro, K., Coto-Montes, A., \& Oliván, M. (2008). Prediction of the fatty acid composition of beef by near infrared transmittance spectroscopy. Meat Science, 78(3), 248-255.

Sikorska, E., Khmelinskii, I., \& Sikorski, M. (2011). Analysis of olive oils by fluorescence spectroscopy: Methods and applications. In D. Boskou (Ed.), Olive oil-constituents, quality, health properties and bioconversions (pp. 63-88). Rijeka: InTech.

Siri-tarino, P. W., Sun, Q., Hu, F. B., \& Krauss, R. M. (2010a). Meta-analysis of prospective cohort studies evaluating the association of saturated fat with cardiovascular disease. American Journal of Clinical Nutrition, 91(3), 535-546.

Siri-tarino, P. W., Sun, Q., Hu, F. B., \& Krauss, R. M. (2010b). Saturated fat, carbohydrate, and cardiovascular disease. American Journal of Clinical Nutrition, 91(5), 502-509.

Skjervold, P., Taylor, R., Wold, J., Berge, P., Abouelkaram, S., Culioli, J., \& Dufour, É. (2003). Development of intrinsic fluorescent multispectral imagery specific for fat, connective tissue, and myofibers in meat. Food Chemistry, 68(4), 1161-1168.

Swatland, H. J. (1987). Autofluorescence of adipose tissue measured with fibre optics. Meat Science, 19(4), 277-284 
Swatland, H., Gullett, E., Hore, T., \& Buttenham, S. (1995). UV fiber-optic probe measurements of connective tissue in beef correlated with taste panel scores for chewiness. Food Research International, 28(1), 23-30.

Tøgersen, G., Arnesen, J. F., Nilsen, B. N., \& Hildrum, K. I. (2003). On-line prediction of chemical composition of semi-frozen ground beef by non-invasive NIR spectroscopy. Meat Science, 63(4), 515-523.

Wahrburg, U. (2004). What are the health effects of fat? European Journal of Nutrition, 43(1), i6-i11.

Williams, P. (2003). Near-infrared technology: Getting the best out of light (1st ed.). Nanaimo: Value Added Wheat CRC, Limited.

Wold, J. P., Kvaal, K., \& Egelandsdal, B. (1999a). Quantification of intramuscular fat content in beef by combining autofluorescence spectra and autofluorescence images. Applied Spectroscopy, 53(4), 448-456.
Wold, J. P., Lundby, F., \& Egelandsdal, B. (1999b). Quantification of connective tissue (hydroxyproline) in ground beef by autofluorescence spectroscopy. Journal of Food Science, 64(3), 377-383.

Wood, J. D., Enser, M., Fisher, A. V., Nute, G. R., Sheard, P. R., Richardson, R. I., Whittington, F. M. (2008). Fat deposition, fatty acid composition and meat quality: A review. Meat Science, 78(4), 343-358.

Yaacoub, R., Saliba, R., Nsouli, B., Khalaf, G., Rizkallah, J., \& Birlouez-Aragon, I. (2009). Rapid assessment of neoformed compounds in nuts and sesame seeds by front-face fluorescence. Food Chemistry, 115(1), 304-312. 